\title{
Continuity of primary care and emergency department utilization among elderly people
}

\author{
Raluca lonescu-Ittu MSc, Jane McCusker MD DrPH, Antonio Ciampi PhD, \\ Alain-Michel Vadeboncoeur MD, Danièle Roberge PhD, Danielle Larouche MSc, Josée Verdon MD MSc, \\ Raynald Pineault MD PhD
}

$\infty \quad$ See related article page $\mathrm{I}_{3} 85$

\section{ABstract}

Background: People aged $6_{5}$ years or more represent a growing group of emergency department users. We investigated whether characteristics of primary care (accessibility and continuity) are associated with emergency department use by elderly people in both urban and rural areas.

Methods: We conducted a cross-sectional study using information for a random sample of 95173 people aged $6_{5}$ years or more drawn from provincial administrative databases in Quebec for 2000 and 2001. We obtained data on the patients' age, sex, comorbidity, rate of emergency department use (number of days on which a visit was made to an amergency department per 1000 days at risk [i.e., alive and not in hospital] during the 2-year study period), use of hospital and ambulatory physician services, residence (urban v. rural), socioeconomic status, access (physician: population ratio, presence of primary physician) and continuity of primary care.

Results: After adjusting for age, sex and comorbidity, we found that an increased rate of emergency department use was associated with lack of a primary physician (adjusted rate ratio $[R R] 1.45,95 \%$ confidence interval $[\mathrm{Cl}]$ 1.41-1.49) and low or medium (v. high) levels of continuity of care with a primary physician (adjusted RR 1.46, 95\% Cl 1.44-1.48, and $1.27,95 \% \mathrm{Cl} 1.25^{-1.29}$, respectively). Other significant predictors of increased use of emergency department services were residence in a rural area, low socioeconomic status and residence in a region with a higher physician:population ratio. Among the patients who had a primary physician, continuity of care had a stronger protective effect in urban than in rural areas.

Interpretation: Having a primary physician and greater continuity of care with this physician are factors associated with decreased emergency department use by elderly people, particularly those living in urban areas.

Une version française de ce résumé est disponible à l'adresse www.cmaj.ca/cgi/content/full/I77/II/I362/DCI

CMAJ 2007;177(II):1362-8

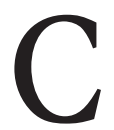
anada is reforming its health care system, with primary care as a major focus. ${ }^{1}$ The population of Canadians aged 65 years or older is expected to double by $2026^{2}$ and already accounts for the largest share of total health care expenditures. ${ }^{3}$ Thus, it is important to evaluate primary care services in this population. Because the emergency department often acts as a safety net for patients receiving inadequate primary care, ${ }^{4}$ emergency department use may be an important indicator of the adequacy of primary care services.

The main determinants of emergency department use by elderly people are the severity and the nature of the medical needs of the patient (overall and specific comorbidities). ${ }^{5}$ After adjustment for need, increased access to and continuity of primary care may also be associated with lower emergency department use. ${ }^{5}$ However, most studies that investigated the impact of access and continuity of primary care were carried out in the United States, where the health care system is fundamentally different from Canada's. ${ }^{5-8}$ Furthermore, most of these studies used selfreported measures of access and continuity of primary care. ${ }^{5,7,9}$

We sought to identify determinants of emergency department use in a population-based sample of elderly people in Quebec, with particular focus on measures of access to and continuity of primary care. Access was defined by 2 measures: (a) presence of a primary physician and (b) physician: population ratio. Relational continuity was defined as the proportion of primary care visits with the primary physician. ${ }^{10,11}$ Finally, because primary care services in Quebec are organized differently in urban and rural areas, ${ }^{12}$ we also compared the association between emergency department use and continuity of care for urban and rural areas.

\section{Methods}

\section{Study design and data sources}

For this cross-sectional population-based study, we used records from provincial administrative databases in Quebec for 2000 and 200I. We obtained individual-level variables from

From the Departments of Epidemiology, Biostatistics and Occupational Health (Ionescu-Ittu, McCusker, Ciampi) and Medicine (Verdon), McGill University, Montréal, Que.; Institut de Cardiologie de Montréal (Vadeboncoeur), Montréal, Que.; Hôpital Charles LeMoyne (Roberge, Larouche), Longueuil, Que.; and Direction de Santé Publique de Montréal (Pineault), Montréal, Que. 
physician claims and prescription drug databases maintained by the Régie de l'assurance maladie du Québec (RAMQ), and the Med-Echo database of hospital discharge information. These 3 databases have complete medical records on all Quebec residents aged 65 years or more. Each subject was assigned a unique identification number by the RAMQ that was used to link information from the 3 databases at the individual level. We determined socioeconomic status by linking the patient's postal code with area of residence using Statistics Canada 200I population census data. ${ }^{13}$ Information on the number of general practitioners and specialists within each of the $\mathrm{I} 6$ health regions of Quebec was obtained from Quebec's Ministère de la Santé et des Services sociaux.

Use of the provincial databases for the study was approved by the provincial Commission on Access to Information and by the institutional review board of the institution where the research was performed. The true identity of the patients was not disclosed to the researchers.

\section{Study sample}

The study sample was drawn from a database used in a previous study ${ }^{14}$ containing information on 892613 Quebec residents aged $6_{5}$ years or more on Jan. I, 2000, who had at least I medical insurance charge in 2000 or 200I. To increase efficiency of the analyses, we selected a simple random sample of Ioo 000 patients with equal probability and no replacement (i.e., all subjects had the same probability of being selected, and each subject could be selected only once). We excluded 4327 people who were in long-term care facilities, I42 who were in hospital on Jan. I, 2000, and died before discharge, and 358 patients whose postal codes were unavailable. The final study sample was 95173 (Figure I).

\section{Measures}

The primary outcome was the rate of emergency department utilization. Over the 2-year study period, a patient may have had more than I emergency department visit, and visits may have lasted I or more consecutive days..$^{15}$ The main determinants of interest were measures of primary care access and continuity of care. Covariates included measures of medical need and sociodemographic characteristics.

A primary care visit was defined as an outpatient visit with a potential primary physician. ${ }^{16,17}$ Given that elderly people with chronic diseases often see specialists regularly and consider them as their primary care physician (especially when there is a shortage of general practitioners), ${ }^{16,17}$ we defined "primary physician" to include the following specialists in addition to general practitioners: internal medicine specialists, allergy specialists, cardiologists, dermatologists, gastroenterologists, obstetrician-gynecologists, hematologists, respirologists, physiatrists, neurologists, nephrologists, endocrinologists, rheumatologists and emergency medicine specialists. We identified the primary physician using an algorithm validated for elderly patients ${ }^{18}$ that takes into account both the physician's specialty and the frequency of visits. The algorithm yields 3 categories of primary physician: general practitioner (for patients who have visited a general practitioner at least once during the study period), specialist (for patients who did not visit a general practitioner but visited specialists during the study period) and no primary physician (for patients who had the same number of visits to more than I general practitioner or specialist).

Continuity of care can be viewed as (a) information transfer, (b) coordination between different providers (information or management continuity) or (c) an ongoing relationship between a patient and a health care provider (relational continuity of care). ${ }^{11}$ In our study, we focused on relational continuity, measured using the usual-provider continuity in$\operatorname{dex}^{10}$ as the proportion of visits with the patient's usual primary physician out of the total number of primary care visits during the study period. We chose this index because its interpretation is straightforward and it is the most widely used measure of relational continuity of care that can be computed using administrative databases. ${ }^{8,11,19}$

Because the usual-provider continuity index is unreliable for people with fewer than 3 primary care visits ${ }^{11}$ and those without a primary physician, we combined it and the measure for type of primary physician into a single variable with 5 categories: high (continuity index $>80 \%$ ), medium (index $>50 \%$ and $\leq 80 \%$ ) or low (index $\leq 50 \%$ ) continuity of care with the primary physician; 3 or more primary care visits but no primary physician; and fewer than 3 primary care visits (low primary care users). In the absence of any known substantive cutoff point for the usual-provider continuity index, we chose points that maximize the statistical efficiency of the analysis (based on the tertile distribution of the index among patients with 3 or more primary care visits).

We grouped the availability of physicians (general practitioners and specialists) in each of $\mathrm{I} 6$ health regions in Quebec into 3 categories: low ( $<$ I general practitioner and < I specialist per 1000 population), mixed ( $\geq$ I general practitioner and $<$ I specialist per Iooo population) and high ( $\geq$ I general practitioner and $\geq$ I specialist per Iooo population). No health region had a mix of $\geq$ I specialist and < I general practitioner per 1000 population.

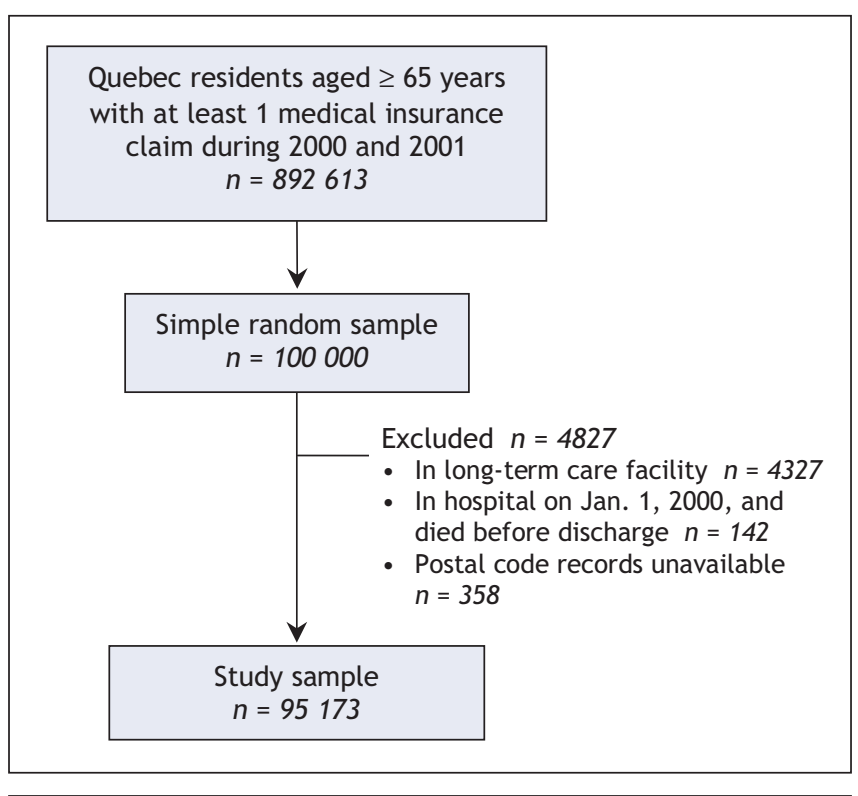

Figure 1: Selection of study sample. 
We used 2 comorbidity indices to measure medical need. The Charlson comorbidity index $\mathrm{x}^{20}$ is based on diagnostic codes from hospital discharge and physician claims data and is calculated by assigning weights to 17 comorbid conditions. The chronic disease score ${ }^{21}$ uses outpatient drug prescriptions and is calculated by assigning weights to 26 medication classes corresponding to 17 comorbidity categories. For both indices, weights are added to determine an overall comorbidity score, with high overall scores $(\geq 6)$ indicating a severe burden of comorbidity. Although there is partial overlap of the comorbidity categories used in each measure (e.g., diabetes, ulcer, tumours), the 2 scores use different sources of administrative data with different limitations; therefore, combining them in the analysis may reduce confounding bias. ${ }^{22}$ In addition to the comorbidity indices to measure medical need, we used 2 specific comorbidities (cardiovascular disease [International Classification of Diseases 9th revision (ICD-9) codes 390.0-459.9] and digestive disease [ICD-9 codes 520.0-579.9]), which have previously been shown to be associated with increased emergency department use, ${ }^{6,23} 2$ measures of health services utilization (total days in hospital and total number of primary care visits over the study period); and death during the study period (a proxy measure of terminal illness or deteriorating health status).

We obtained sociodemographic data on age, sex, socioeconomic status, area of residence and proximity to an emergency department. We measured socioeconomic status with validated ecologic measures of material and social deprivation, ${ }^{24}$ grouped into 3 categories: high, medium and low. The method matches the patient's postal code with census data on 6 indicators of material and social deprivation (education, employment, income, marital status, single parenting and living alone) to create an aggregate ecologic measure of material and social deprivation. We classified area of residence as urban (corresponding to census metropolitan area or urban core categories in Statistics Canada classification ${ }^{13}$ ), rural (remaining categories), and intermediate or mixed (when the first 3 digits of the postal code corresponded to both urban and rural categories). We defined proximity to an emergency department as the presence of an emergency department in the area served by the patient's community health centre (in Quebec, 95 community health centres provide health and social services to residents of defined geographic areas).

\section{Statistical analysis}

Emergency department utilization during the study period was described as a rate (number of days on which a visit was made to an emergency department per Iooo days at risk [i.e., days when the person was alive and not in hospital]). We used the individual patient as the unit of analysis. In comparing characteristics of patients, we reported medians and interquartile ranges for continuous variables and proportions for categorical variables. We used the Wilcoxon rank sum test to test for equality of medians for age, comorbidity scores, days in hospital and number of primary care visits, and the $\chi^{2}$ test to test for equality of proportions for sex, underlying comorbidity, residence, socioeconomic status, access and level of continuity of primary care.

We assessed the unadjusted and adjusted relation between each independent variable and the rate of emergency depart- ment use using Poisson regression analysis, from which we report the rate ratios and $95 \%$ confidence intervals. We decided a priori to keep in the final model the variables age, sex and continuity of care with the primary physician, regardless of their statistical significance. However, given our large sample, it was not necessary to exclude any variables from the model based on statistical significance.

We tested for collinearity by checking the correlations between variables and the variance inflation factor. ${ }^{25} \mathrm{We}$ excluded total number of primary care visits from the multivariable analysis because of collinearity problems (people with fewer than 3 primary care visits represented the "low primary care users" category of the variable describing continuity of care with the primary physician). However, we performed a sensitivity analysis involving patients with 3 or more primary care visits, in which we included both the total number of primary care visits and the continuity of care with the primary physician as variables in the multivariable model. We also tested for interactions between area of residence and continuity of care with the primary physician. If the interaction term was significant at $p<0.05$, we reran the multivariable model stratified by location of residence (urban or rural) to explore in more detail how the effect of continuity of care with the primary physician differed in these 2 areas.

Where possible, "missing" was included as a separate category in the model if data were unavailable. However, we had to exclude $35^{8}$ people whose postal code records were missing, because the postal codes were necessary to calculate several measures of access to care.

\section{Results}

\section{Study population}

Over the 2-year study period, $52 \%$ of the study population did not use emergency department services, $18 \%$ used the emergency department I day, $\mathrm{II} \% 2$ days and the remaining $\mathrm{I} 8 \%$ $\geq 3$ days (Table $\mathrm{I}$ ). The average rate of emergency department use was 2.I4 days per Iooo days at risk. We were able to identify a primary physician for $83 \%$ of the patients in the sample; of these, $36 \%$ had high relational continuity of care with that physician $(\geq 80 \%$ of their total primary care visits were with the primary physician). For $79 \%$ of the sample, the primary physician was a general practitioner. The mean usual-provider continuity index among patients with a primary physician was $66 \%$ (median $67 \%$ ). The median number of primary care visits among patients with a primary physician was II (interquartile range $7-\mathrm{I} 8$ ), as compared with 7 (interquartile range 4-I2) among those without a primary physician and I (interquartile range 0-2) among low primary care users (those with fewer than 3 primary care visits).

\section{Predictors of emergency department use}

Low and medium levels of continuity of care with the patient's primary physician and lack of a primary physician were factors associated with an increased rate of emergency department use (Table 2). Furthermore, there was a doseresponse relation between continuity of care and emergency department use among patients with a primary physician. Other independent predictors of increased emergency depart- 
ment use were high overall comorbidity, presence of cardiovascular or digestive disease, increased number of days in hospital, terminal illness (or deteriorating health), higher age, living in a rural area, low socioeconomic status, living near an emergency department and living in a region with greater availability of general practitioners.

\section{Interaction between area of residence} and continuity of care with the primary physician

The formal tests for interaction between area of residence and continuity of care with the primary physician yielded statistically significant results $(p<0.0 \mathrm{r})$ when added to the multivariable model, which prompted us to perform separate multivariable analyses for patients living in urban and rural areas. The analyses revealed that the rate ratios associated with low and medium levels of continuity of care and being a low primary care user (v. high continuity of care) were higher in urban than in rural areas. Specifically, the adjusted rate ratios in urban areas were I. 58 (95\% confidence interval [CI] I.55-I.6I) for low continuity of care, I.3I (95\% CI I.28-I.33) for medium continuity of care and I.42 (95\% CI I.38-I.46) for low primary care user. The corresponding rates in rural areas were I.28 (95\% CI I.22-I.36), I.29

Table 1: Characteristics of the study sample of 95173 Quebec residents aged 65 or more who had at least 1 medical insurance claim from Jan. 1, 2000, to Dec. 31, 2001, by emergency department use

\begin{tabular}{|c|c|c|c|c|}
\hline \multirow[b]{2}{*}{ Characteristic } & \multirow[b]{2}{*}{$\begin{array}{c}\text { Total } \\
n=95173\end{array}$} & \multicolumn{2}{|c|}{$\begin{array}{l}\text { Visited emergency department in study } \\
\text { period; no. (\%) of patients* }\end{array}$} & \multirow[b]{2}{*}{$p$ value } \\
\hline & & $\begin{aligned} \text { Yes } & \\
n= & 45886\end{aligned}$ & $\begin{aligned} & \text { No } \\
n= & 49287\end{aligned}$ & \\
\hline Age, yr, median (IQR) & $74(69-79)$ & $75(70-80)$ & $73(69-78)$ & $<0.001$ \\
\hline Male sex, no. (\%) & $39230(41)$ & $19275(42)$ & $19955(40)$ & $<0.001$ \\
\hline Charlson comorbidity score, median (IQR) $\dagger$ & $1(0-2)$ & $2(0-4)$ & $0(0-2)$ & $<0.001$ \\
\hline $\begin{array}{l}\text { Medication-based chronic disease score, } \\
\text { median (IQR)‡ }\end{array}$ & $6(1-10)$ & $7(3-11)$ & $4(0-8)$ & $<0.001$ \\
\hline Cardiovascular disease & $63733(67)$ & $34848(76)$ & 28885 (59) & $<0.001$ \\
\hline Digestive disease & $26120(27)$ & $17006(37)$ & $9114(18)$ & $<0.001$ \\
\hline Terminal illness & $6731 \quad(7)$ & $5979(13)$ & $752(1)$ & $<0.001$ \\
\hline Days in hospital, median (IQR) & $0(0-3)$ & $2(0-15)$ & $0(0-0)$ & $<0.001$ \\
\hline Primary care visits, median (IQR) & $10(5-16)$ & $12(6-20)$ & $8(4-14)$ & $<0.001$ \\
\hline Level of continuity of care with primary physician & & & & $<0.001$ \\
\hline High & $28320(30)$ & $11700(25)$ & $16620(34)$ & \\
\hline Medium & $30068(32)$ & $15070(33)$ & $14998(30)$ & \\
\hline Low & $20410(21)$ & $11859(26)$ & $8551(17)$ & \\
\hline No primary physician & 3803 & 1928 & 1875 & \\
\hline Low primary care user (<3 visits) & $12572(13)$ & $5329(12)$ & $7243(15)$ & \\
\hline Area of residence & & & & $<0.001$ \\
\hline Urban & $66198(70)$ & 30679 (67) & $35519(72)$ & \\
\hline Intermediate (mixed) & $22741(24)$ & $11702(25)$ & $11039(22)$ & \\
\hline Rural & $6234 \quad(6)$ & $3505 \quad(8)$ & $2729 \quad(6)$ & \\
\hline Socioeconomic status & & & & $<0.001$ \\
\hline Low & $18714(20)$ & $9455(21)$ & $9259(19)$ & \\
\hline Medium & $59139(62)$ & $27702(60)$ & $31437(64)$ & \\
\hline High & $8418 \quad(9)$ & $3368 \quad(7)$ & $5050(10)$ & \\
\hline Missing data & $8902 \quad(9)$ & $5361(12)$ & $3541 \quad(7)$ & \\
\hline Residence near an emergency department & $61311(64)$ & $31297(68)$ & $30014(61)$ & $<0.001$ \\
\hline Physician availability in area of residence & & & & $<0.001$ \\
\hline Low & 47072 (49) & 22357 (49) & $24715(50)$ & \\
\hline Mixed§ & $7257 \quad(8)$ & 4290 & $2967 \quad(6)$ & \\
\hline High & $40844(43)$ & $19239(42)$ & $21605(44)$ & \\
\hline
\end{tabular}

Note: $I Q R$ = interquartile range.

*Unless stated otherwise.

†Maximum score 20; higher values show higher overall comorbidity.

¥Maximum score 29; higher values show higher comorbidity.

§High availability of general practitioners, low availability of specialists. 
(95\% CI I.24-I.34) and I.09 (95\% CI I.02-I.I6). However, the effect of the lack of a primary physician (v. high continuity of care) was stronger in rural (rate ratio I.70, 95\% CI I.55-I.86) than in urban areas (rate ratio I.57, 95\% CI I.55-I.6I).

\section{Sensitivity analysis}

Sensitivity analysis among patients with 3 or more primary care visits was used to investigate the impact of including the total number of primary care visits in the multivariable model. The results showed that the magnitude and direction of the effect of continuity of care with the primary physician (with high continuity of care as the reference category) did not change after including total number of primary care visits in the analysis: rate ratio $\mathrm{I} .27$ (95\% CI I.25-I.29) for medium continuity of care; I.43 (95\% CI I.4I-I.46) for low continuity of care and I.46 (95\% CI I.43-I.5I) for lack of a primary physician. Furthermore, the number of primary care visits had no independent effect on overall emergency department use (rate ratio I.00, 95\% CI I.00-I.00).

\section{Interpretation}

After adjusting for measures of medical need, demographic characteristics and other covariates, we found that increased

Table 2: Predictors of emergency department use* identified by Poisson regression analysis

\begin{tabular}{|c|c|c|c|c|}
\hline Factor & $\begin{array}{l}\text { Unadjusted rate } \\
\text { ratio }(95 \% \mathrm{Cl})\end{array}$ & $p$ value & $\begin{array}{l}\text { Adjusted rate ratio } \\
\qquad(95 \% \mathrm{Cl})\end{array}$ & $p$ value \\
\hline Age $\neq$ & $1.42(1.41-1.43)$ & $<0.001$ & $1.18(1.17-1.18)$ & $<0.001$ \\
\hline Male sex & $1.11(1.10-1.12)$ & $<0.001$ & $1.06(1.05-1.07)$ & $<0.001$ \\
\hline Charlson comorbidity score§ & $1.21(1.20-1.21)$ & $<0.001$ & $1.07(1.07-1.07)$ & $<0.001$ \\
\hline Medication-based chronic disease score§ & $1.10(1.10-1.10)$ & $<0.001$ & $1.04(1.04-1.05)$ & $<0.001$ \\
\hline Cardiovascular disease & $2.31(2.28-2.34)$ & $<0.001$ & $1.41(1.39-1.44)$ & $<0.001$ \\
\hline Digestive disease & $2.29(2.27-2.31)$ & $<0.001$ & $1.66(1.64-1.68)$ & $<0.001$ \\
\hline Terminal illness & $4.96(4.89-5.04)$ & $<0.001$ & $2.01(1.98-2.05)$ & $<0.001$ \\
\hline Days in hospitalף & $1.08(1.08-1.08)$ & $<0.001$ & $1.05(1.05-1.05)$ & $<0.001$ \\
\hline \multicolumn{5}{|l|}{$\begin{array}{l}\text { Level of continuity of care } \\
\text { with primary physician }\end{array}$} \\
\hline $\mathrm{High}^{* *}$ & 1.00 & & 1.00 & \\
\hline Medium & $1.37(1.35-1.39)$ & $<0.001$ & $1.27(1.25-1.29)$ & $<0.001$ \\
\hline Low & $1.83(1.81-1.86)$ & $<0.001$ & $1.46(1.44-1.48)$ & $<0.001$ \\
\hline No primary physician & $1.46(1.42-1.49)$ & $<0.001$ & $1.45(1.41-1.49)$ & $<0.001$ \\
\hline Low primary care user (<3 visits) & $0.97(0.95-0.99)$ & $<0.002$ & $1.24(1.21-1.27)$ & $<0.001$ \\
\hline \multicolumn{5}{|l|}{ Area of residence } \\
\hline Urban $^{* *}$ & 1.00 & & 1.00 & \\
\hline Intermediate (mixed) & $1.14(1.13-1.16)$ & $<0.001$ & $1.22(1.20-1.23)$ & $<0.001$ \\
\hline Rural & $1.51(1.49-1.54)$ & $<0.001$ & $1.51(1.48-1.54)$ & $<0.001$ \\
\hline \multicolumn{5}{|l|}{ Socioeconomic status } \\
\hline High** $^{* *}$ & 1.00 & & 1.00 & \\
\hline Medium & $1.28(1.25-1.30)$ & $<0.001$ & $1.12(1.10-1.15)$ & $<0.001$ \\
\hline Low & $1.50(1.46-1.53)$ & $<0.001$ & $1.25(1.22-1.27)$ & $<0.001$ \\
\hline Missing data & $2.12(2.07-2.18)$ & $<0.001$ & $1.50(1.46-1.54)$ & $<0.001$ \\
\hline Residence near an emergency department & $1.27(1.25-1.28)$ & $<0.001$ & $1.21(1.19-1.22)$ & $<0.001$ \\
\hline \multicolumn{5}{|l|}{ Physician availability in region of residence } \\
\hline Low $^{* *}$ & 1.00 & & 1.00 & \\
\hline Mixed†† & $1.44(1.41-1.46)$ & $<0.001$ & $1.23(1.21-1.26)$ & $<0.001$ \\
\hline High & $1.05(1.04-1.06)$ & $<0.001$ & $1.10(1.08-1.11)$ & $<0.001$ \\
\hline
\end{tabular}

Note: $\mathrm{Cl}=$ confidence interval.

${ }^{*}$ Rate of use calculated as number of days on which a visit was made to an emergency department per 1000 days at risk (i.e., alive and not in hospital).

†Adjusted for all other variables presented in the table.

$\neq$ Change in rate of emergency department use per decade increase in age.

$\S$ Change in rate of emergency department use per point increase in comorobidity score.

IChange in rate of emergency department use per 5-day increase in total number of days in hospital.

**Reference category.

††High availability of general practitioners, low availability of specialists. 
emergency department use by elderly patients was associated with lack of a primary physician, lower continuity of care with the primary physician, low overall use of primary care services and residence in a region with more general practitioners per Iooo population. Furthermore, lower continuity of care and low overall use of primary care services appeared to have a stronger effect on emergency department use among urban than among rural residents, whereas absence of a primary physician had a stronger effect among rural residents. The associations between emergency department use and the individual-level measures of primary care access and continuity in our study are similar to those reported in several studies in the United States using different measures of these constructs in elderly populations $\mathrm{s}^{7-9,26}$ and in other studies in younger populations (the general population in the United States ${ }^{27}$ and the United Kingdom ${ }^{28}$ and patients with terminal cancer in Canada ${ }^{29}$ ).

Because this is a cross-sectional study, we cannot determine the direction of the observed associations. For example, a continuous relationship with a primary physician may plausibly reduce emergency department use, whereas elderly people who prefer to receive primary care services in an emergency department may be less likely to seek and maintain a relationship with a primary physician. Although the former explanation is consistent with our finding that there was a dose-effect relation between continuity of care and emergency department use, the latter explanation is consistent with our finding that low primary care users (those with fewer than 3 primary care visits) were more likely than those with high continuity of care to use emergency department services. This suggests that continuity of care may have a beneficial effect only among elderly people who seek and develop a relationship with a primary physician. Indeed, continuity of care is likely to affect decision-making for both the patient and the physician. ${ }^{27}$ An ongoing relationship with a patient allows the physician to acquire knowledge, not only about the patient's medical problems, but also about his or her attitudes and values. A patient with an ongoing relationship with a physician is likely to develop trust in the physician's expertise and medical judgment and to ask the physician for advice before going to an emergency department with a medical problem he or she perceives as urgent.

Although the observed association between continuity of care among patients with a primary physician and emergency department use could also be explained by a protective effect of primary care services in general, we found a low correlation between the usual provider continuity index and total number of primary care visits; also, adding total number of primary care visits to the sensitivity analysis model did not change the effect of continuity of care, which suggests that continuity of care has a protective effect above and beyond that of primary care utilization.

Finally, the different relation between continuity of care and emergency department use in rural and urban areas underlines differences in primary care organization in urban and rural areas of Quebec. In rural areas, primary physicians are more likely than primary physicians in urban areas to practise in networks that include both private practices and emergency departments and, therefore, are more likely to schedule visits with their patients in the emergency department. ${ }^{12}$ We also found that a greater number of general practitioners alone may not increase access to primary care or continuity of care; thus, interventions to decrease emergency department use should go beyond increasing numbers of physicians and include such organizational measures as evening and weekend coverage in general practitioner practices.

Our study has several limitations. First, comorbidity as measured by the Charlson comorbidity inde ${ }^{20}$ may be underestimated in elderly patients using fewer services. ${ }^{30}$ Second, the medical claims data of the Régie de l'assurance maladie du Québec miss about I0.5\% of medical services provided by salaried physicians. ${ }^{31}$ Third, potentially important factors, such as perceived health status, health beliefs, race and ethnic background, were not available. Fourth, we used a measure of socioeconomic status that may result in an ecological fallacy. Fifth, we used an indirect measure of continuity of care based on health utilization rates, because this study was based exclusively on data from administrative databases and we were unable to ask patients to identify their primary physician and report how long they have been seeing that physician.

In conclusion, our study shows that having a primary physician and a high level of continuity of care was associated with decreased emergency department use. This finding underlines the importance of research into alternative primary care organizational models in Canada.

This article has been peer reviewed.

Competing interests: None declared.

Contributors: Raluca Ionescu-Ittu was responsible for the concept and design of the study, data analysis and drafting of the manuscript. Jane McCusker was responsible for data acquisition and contributed to the concept and design of the study, interpretation of the data and critical review of the manuscript for methodological content and accuracy. Antonio Ciampi contributed to the study design, data analysis and revision of the manuscript. Alain Vadeboncoeur contributed to acquisition and interpretation of the data and revision of the manuscript. Danièle Roberge, Danielle Larouche, Josée Verdon and Raynald Pineault contributed to interpretation of the data and revision of the manuscript. All of the authors gave final approval of the version to be published.

Acknowledgements: We thank Eric Belzile for his assistance with the data analysis.

This research was supported by an operating grant from the Canadian Institutes of Health Research (grant no. IAP 62346, principal investigator Jane McCusker). Raluca Ionescu-Ittu was supported by a scholarship award from the Groupe interuniversitaire de recherche sur les urgences.

\section{REFERENCES}

I. Canadian Institute for Health Information. Health care in Canada, 2003. Ottawa: Statistics Canada; 2003. Available: secure.cihi.ca/cihiweb/dispPage.jsp?cw _page=AR_43_E (accessed 2007 Oct 9).

2. Canada's seniors: a growing population. Ottawa: Health Canada; 200I. Available: www.hc-sc.gc.ca/seniors-aines/pubs/factoids/200I/pdf/I-37_e.pdf (accessed 2007 Oct 9).

3. National Advisory Council on Ageing. Aging vignettes \#21-33: a quick portrait on Canadian health and seniors. Ottawa: Health Canada; 1996.

4. Billings J, Parikh N, Mijanovich T. Emergency department use in New York City: a substitute for primary care? New York: Commonwealth Fund Publications; 2000.

5. McCusker J, Karp I, Cardin S, et al. Determinants of emergency department visits by older adults: a systematic review. Acad Emerg Med 2003;10:1362-70.

6. Bazargan M, Bazargan S, Baker RS. Emergency department utilization, hospital admissions, and physicians visits among elderly African-American persons. Gerontologist 1998;38:25-36.

7. Lishner DM, Rosenblatt RA, Baldwin LM, et al. Emergency department use by the rural elderly. J Emerg Med 2000;18:289-97. 
8. Rosenblatt RA, Wright GE, Baldwin LM, et al. The effect of the doctor-patient relationship on emergency department use among the elderly. Am J Public Health 2000;90:97-102.

9. Weiss LJ, Blustein J. Faithful patients: the effect of long-term physician-patient relationships on the costs and use of health care by older Americans. Am J Public Health $1996 ; 86: 1742-7$.

ro. Breslau N, Reeb KG. Continuity of care in a university-based practice. J Med Educ I975;50:965-9.

II. Reid R, Haggerty J, McKendry R. Defusing the confusion: concepts and measures of continuity of healthcare. Ottawa: Canadian Health Services Research Foundation, the Canadian Institute for Health Information, and the Advisory Committee on Health Services of the Federal/Provincial/Territorial Deputy Ministers of Health; 2002.

I2. Pineault $\mathrm{R}$, Tousignant $\mathrm{P}$, Roberge $\mathrm{D}$, et al. Research collective on the organization of primary care services in Quebec: summary report. Montréal: Direction de sante publique, Agence de développement de réseaux locaux de services de santé et de services sociaux de Montréal; 2005. Available: www.chsrf.ca/research_themes/pdf /summary_report_e.pdf (accessed 2007 Oct 9).

I3. 2001 census dictionary. Ottawa: Statistics Canada; 2003. Cat no 92-378-XIE.

I4. McCusker J, Ionescu-Ittu R, Ciampi A, et al. Hospital characteristics and emergency department care of older patients are associated with return visits. Acad Emerg Med 2007;14:426-33.

I5. Roberge $\mathrm{D}$, Larouche $\mathrm{D}$, Pineault $\mathrm{R}$, et al. Les unités d'urgence hospitalières dans un réseau en transformation: l'expérience Montréalaise. Gestion hospitalières 2002;402:706-10.

I6. Goldberg HI, Dietrich AJ. The continuity of care provided to primary care patients. A comparison of family physicians, general internists and medical subspecialists. Med Care $1985 ; 23: 63-73$.

I7. Aiken LH, Lewis CE, Craig J, et al. The contribution of specialists to the delivery of primary care. NEngI J Med I979;300:1363-70.

I8. McCusker J, Dendukuri N, Tousignant P, et al. Rapid two-stage emergency department intervention for seniors: impact on continuity of care. Acad Emerg Med 2003;10:233-43

I9. Gill JM, Mainous III AG. The role of provider of continuity in preventing hospitalizations. Arch Fam Med I998;7:352-7.

20. D'Hoore W, Bouckaert A, Tilquin C. Practical considerations on the use of the Charlson comorbidity index with administrative data bases. J Clin Epidemio 1996;49:1429-33.
2I. Von Korff M, Wagner EH, Saunders K. A chronic disease score from automated pharmacy data. JClin Epidemiol 1992;45:197-203.

22. Schneeweiss S, Wang PS, Avorn J, et al. Consistency of performance ranking of comorbidity adjustment scores in Canadian and US utilization data. J Gen Intern Med 2004;ig(5 pt I):444-50.

23. McCusker J, Cardin S, Bellavance F, et al. Return to the emergency department among elders: patterns and predictors. Acad Emerg Med 2000;7:249-59.

24. Pampalon R, Raymond G. A deprivation index for health and welfare planning in Quebec. Chronic Dis Can 2000;2I:104-I3.

25. Freund RJ, Wilson WJ. Diagnosing multicollinearity. In: Regression analysis: statistical modeling of a response variable. Ist ed. San Diego: Academic Press; 1997. p. 192-5

26. Wolinsky FD, Coe RM, Miller DK, et al. Health services utilization among the noninstitutionalized elderly. J Health Soc Behav I983;24:325-37.

27. Gill JM, Mainous III AG, Nsereko $M$. The effect of continuity of care on emergency department use. Arch Fam Med 2000;9:333-8.

28. Sweeney KG, Gray DP. Patients who do not receive continuity of care from their general practitioner: Are they a vulnerable group? BrJ Gen Pract 1995;45:133-5.

29. Burge F, Lawson B, Johnston G. Family physician continuity of care and emergency department use in end-of-life cancer care. Med Care 2003;4II:992-IO0I.

30. Quam L. Using claims data for epidemiologic research. The concordance of claims-based criteria with the medical record and patient survey for identifying a hypertensive population. Med Care 1993;31:498-507.

3I. Régie de l'assurance maladie du Québec. Coût et nombre de services médicaux, nombre de médecins, montant moyen versé, nombre moyen de services par médecin et coût moyen par service selon le mode de rémunération [Tableau 2.03]. Available: https://www.prod.ramq.gouv.qc.ca/IST/CD/CDF_DifsnInfoStats/CDFI_Cnsul InfoStatsCNC_iut/RappPDF.aspx?TypeImpression=pdf\&NomPdf=CBBIRo2A_SMo3 200I_0_O.pdf (accessed 2007 Oct 9).

Correspondence to: Dr. Jane McCusker, Department of Clinical Epidemiology and Community Studies, St. Mary's Hospital Center, 3830 Lacombe Ave., Montréal QC H3T IM5; fax 514 734-2652; jane.mccusker@mcgill.ca

\section{Canadian Medical Association}

\section{Special Awards - Call for Nominations}

Association médicale canadienne

The Canadian Medical Association invites nominations for the 2008 special awards.

- Medal of Honour

- F.N.G. Starr Award

- Medal of Service

- May Cohen Award for Women Mentors

- Sir Charles Tupper Award for Political Action

- Award for Excellence in Health Promotion

- Award for Young Leaders

- Dr. William Marsden Award in Medical Ethics

Refer to the "Awards from CMA" section on cma.ca for detailed criteria on each of the awards or contact the awards co-ordinator at $800663-7336 \times 2280$.

Nominations should be submitted to:

Chair, Committee on Archives and Awards c/o Committee Co-ordinator

Corporate Affairs

Canadian Medical Association

1867 Alta Vista Dr.

Ottawa ON K1G 3 Y6

Closing date for receipt of nominations is Nov. 30, 2007.
L'Association médicale canadienne sollicite des candidatures à ses prix spéciaux pour l'an 2008.

- Médaille d'honneur

- Prix F.N.G. Starr

- Médaille de service

- Prix May-Cohen pour femmes mentors

- Prix Sir-Charles-Tupper d'action politique

- Prix d'excellence en promotion de la santé

- Prix des jeunes chefs de file

- Prix Dr-William-Marsden d'éthique médicale

Voir «Prix et distinctions de l'AMC» sur le site amc.ca pour les critères détaillés de chaque prix ou contacter la coordonnatrice des prix au $800663-7336$, poste 2280 .

Les candidatures doivent être soumises à la :

Présidente, Comité des archives et des distinctions a/s Coordonnatrice des comités

Affaires générale

Association médicale canadienne

1867, promenade Alta Vista

Ottawa (Ontario) K1G 3 Y6

Les candidatures doivent être présentées au plus tard le 30 novembre 2007.

\section{AsSOCIATION P CANADIAN \\ MÉDICALE \& MEDICAL \\ CANADIENNE ASSOCIATION}

